\title{
An Unusual Progression of Follicular Lymphoma Mimicking T-Cell and Histiocyte-Rich Large B-Cell Lymphoma
}

\author{
Ashwini Nargund ${ }^{1,2}$, Steven J. Eastep ${ }^{3}$, Roger A. Warnke ${ }^{1}$ and Yasodha Natkunam ${ }^{1}$ \\ ${ }^{1}$ Department of Pathology, Stanford University School of Medicine, Stanford, CA, USA \\ ${ }^{2}$ Department of Pathology, St John's Medical College and Hospital, Bangalore, India \\ ${ }^{3}$ St. Luke's Hospital, Duluth, Minnesota, USA
}

"Corresponding author: Yasodha Natkunam, Department of Pathology, L235, Stanford University School of Medicine, 300 Pasteur Drive, Stanford, CA 94305-5324, USA, Tel: (650) 725-9354; Fax: (650) 725-7409; E-mail: yaso@stanford.edu

Rec date: Oct 13, 2014, Acc date: Nov 01, 2014, Pub date: Nov 03, 2014

Copyright: (C) 2014 Nargund A, et al. This is an open-access article distributed under the terms of the Creative Commons Attribution License, which permits unrestricted use, distribution, and reproduction in any medium, provided the original author and source are credited.

\begin{abstract}
A significant proportion of follicular lymphoma transform to a higher-grade lymphoma, most commonly, diffuse large B-cell lymphoma. This histologic transformation usually shows diffuse sheets of large cells; however, variant morphologic patterns are also known to occur. We report a case of a 70-year old woman who presented with an unusual large cell transformation of follicular lymphoma with a T-cell and histiocyte-rich background and limited foci of confluent large B-cells. The atypical growth pattern in the excised lymph node led to a broad differential diagnosis that not only included diffuse large B-cell lymphoma but also T-cell lymphoma and nodular lymphocyte predominant Hodgkin lymphoma. Ancillary studies confirmed a neoplastic and clonal B-cell process and immunochemotherapy was initiated. This case illustrates the need for recognition of variant morphologic patterns in large cell transformation of follicular lymphoma for appropriate classification and clinical management.
\end{abstract}

Keywords: Diffuse large B cell lymphoma; Transformation; Progression; T-cell and histiocyte-rich large B-cell lymphoma; Nodular lymphocyte predominant Hodgkin Lymphoma

\section{Introduction}

Follicular lymphoma (FL) is the second most common lymphoma that affects adults in the Western hemisphere. In $15-32 \%$ of patients with FL, progression to a more aggressive lymphoma is known to occur and is associated with an adverse prognosis despite currently available multimodality therapies [1-6]. Histologically, most cases of FL progress to diffuse large B-cell lymphoma (DLBCL), although we and others have reported the rare occurrence of a blastic transformation resembling a B cell lymphoblastic leukemia [7-15] In addition, classical Hodgkin lymphoma (CHL) that can precede or follow FL, as well as composite lymphomas that consist of simultaneous involvement of $\mathrm{CHL}$ and $\mathrm{FL}$, have been reported [16-18].

We describe an unusual case in which atypical B-cell nodules were found in association with an intrafollicular expansion that was rich in T-cells and histiocytes, with only scattered small foci of confluent large B-cells. This case raised differential diagnostic considerations that included a nodular lymphocyte predominant Hodgkin lymphoma (NLPHL), a T-cell and histiocyte-rich large B-cell lymphoma (TCRLBCL), a large B-cell lymphoma arising in the setting of an underlying mature T-cell lymphoma such as an angioimmunoblastic (AITL) or peripheral T-cell lymphoma, not otherwise specified (PTCL, NOS), and an unusual progression of FL. Since the clinical management of these diagnostic considerations are vastly different, it was important to distinguish among these possibilities in an effort to establish a final diagnosis.

\section{Case Report}

The patient was a 70-year old woman who presented with right cervical lymphadenopathy of one-month duration. She reported a 14pound weight loss over the prior several months but denied fevers, chills, or night sweats. In 2001, she had an episode of fever, chills, night sweats and unexplained weight loss with inguinal and axillary lymphadenopathy. An inguinal lymph node biopsy performed at that time was interpreted as follicular hyperplasia. She did not undergo further treatment and remained free of symptoms during the intervening twelve years.

Her past medical history was notable for hypertension, hypothyroidism, osteoporosis, vitamin B12 deficiency, iron deficiency, hiatal hernia, Raynaud's phenomenon with positive anti-nuclear antibodies (ANA), shingles, hyperlipidemia, and peripheral vascular disease with claudication, left greater than right. She was a nonsmoker with moderate alcohol use.

Physical examination revealed an easily visible enlarged lymph node below the angle of the mandible in the right cervical chain estimated at $1.5 \mathrm{~cm}$, multiple small, enlarged lymph nodes in the left axilla and a large palpable lymph node in the right axilla. In June 2013, an excisional biopsy of the large right axillary lymph node was performed, which measured $4 \times 3.5 \times 1.5 \mathrm{~cm}$.

Chest and abdominal CT scans performed for staging revealed superior right mediastinal, bilateral axillary, periaortic, and bilateral inguinal lymphadenopathy, which was radiologically felt to be consistent with lymphoma. There was also a soft tissue mass measuring $2 \mathrm{~cm}$, which expanded the anterior left 4 th rib. Minimal splenomegaly ( $14 \mathrm{~cm}$ with a $1.3 \mathrm{~cm}$ low attenuation lesion) was noted. A positron emission tomography (PET scan) revealed extensive involvement of lymph nodes as well as lytic lesions of the right and left frontal bones, occiput and multiple ribs. The PET report also noted 
bilateral cervical, bilateral axillary, superior anterior mediastinal, common iliac, bilateral external iliac and bilateral inguinal lymphadenopathy with bulky lymphadenopathy of the lumbar retroperitoneum measuring up to $6.4 \mathrm{~cm}$. Focal involvement of the spleen was also detected. The staging bone marrow biopsy revealed no evidence of involvement by lymphoma.

\section{Ancillary Studies}

Immunohistochemistry, in situ hybridization for EBV, fluorescence in situ hybridization (FISH) and polymerase chain reaction (PCR) for $\mathrm{B}$ and $\mathrm{T}$ cell gene rearrangements were performed as previously described [18]. EBV encoded small RNA was detected by in situ hybridization using a Ventana Benchmark instrument (Roche/ Ventana Medical Systems). PCR was performed using BIOMED-2 primers to detect rearrangements of IGH, IGK, TRG and TRB followed by differential fluorescence detection using GeneScan analysis (Life Technologies, Foster City, CA). For FISH, LSI BCL2 Dual Color Break Apart Rearrangement Probe (Vysis, Downers Grove, IL, USA) was used to detect the BCL2 rearrangement. A positive case was defined when the mean number of positive tiles detected was 3 standard deviations above the mean of a negative control (reactive lymphoid tissue). The threshold established was $1.8 \%$ for BCL2 (the mean of the negative control group was $0.65 \pm 0.38$ ).

\section{Results}

\section{Histologic findings}

The lymph node biopsy from 2001 was reviewed and showed wellpreserved overall architecture with vascular transformation of sinuses. The follicles were dispersed and exhibited intact mantle zones; however they appeared to lack polarization and tingible body macrophages. The cellular composition was consistent with a diagnosis of a low-grade (grades 1-2 of 3) follicular lymphoma (Figure 1A-1C). Unfortunately, no flow cytometry or immunohistologic studies were performed at the time of that biopsy, the cell block availability time ( $>10$ years) had lapsed, and only a single H\&E-stained slide was available from the original facility for our review.

The axillary lymph node removed in 2013 measured $4 \mathrm{~cm}$ in maximum dimension and exhibited a homogenous and fleshy cut surface. Histologically, there was partial architectural effacement with atypical follicles interspersed by somewhat expanded interfollicular areas rich in small lymphoid cells, histiocytes, including epithelioid histiocytes, and scattered plasma cells. A proliferation of atypical large cells was sprinkled in the interfollicular areas (Figure 1D-1F). The majority of the atypical follicles (90\%) showed a predominance of small to medium sized cells characteristic of grades 1-2 FL, whereas the remaining follicles showed a cell composition characteristic of grade 3A FL (Figure 1G-1I). In selected follicles, atypical large cells appeared to colonize follicles as well as invade mantle zones. Focally, these large cells were also present in sheets $(<1 \%$ of the total area of the lymph node). Cytologic features of these atypical large cells included nuclear pleomorphism, vesicular chromatin and one to several conspicuous eosinophilic nucleoli. Some of the cells were multinucleated and resembled Reed-Sternberg cells (Figure 1I).

\section{Immunohistologic findings}

Immunohistologic stains for CD20 and CD79a highlighted atypical cells within follicles, mantle zones, interfollicular areas and in focal sheets of large cells (Figure 2A-2F). Immunostains for CD3 and CD5 showed that there were abundant reactive interfollicular small T-cells. B-cell transcription factors OCT2 and BOB1 showed intense expression comparable to background reactive $\mathrm{B}$-cells. The follicles and diffuse areas were strongly positive for BCL2 and weakly positive for the germinal center marker, HGAL (Figure 2B, 2C, 2E and 2F). Interfollicular areas are studded with atypical large B-cells highlighted by CD20 in a TCRLBCL-like areas (Figure 2G). Ki67 highlights a low proliferation in grade 1-2 follicular lymphoma (Figure $2 \mathrm{H}$ ), however in confluent foci of atypical large cells an increased growth fraction (Figure 2I-2J) (Table 1).

The stain for CD21 showed intact follicular dendritic meshworks, although many disrupted follicles were also evident. CD68 stained numerous histiocytes in the interfollicular areas. Assessment for Kappa and lambda light chains by immunohistochemistry as well as in situ hybridization highlighted polytypic plasma cells. Staining for CD10, CD30, CD15, ALK1, LMO2, BCL6, EBV EBER and EBV LMP1 were negative in the atypical large cells (Figure $2 \mathrm{~K}-2 \mathrm{~L}$ ), although rare positive cells (internal positive controls) were present for all stains. PD-1 showed no ringing around the large cells. Ki-67 labelling was seen in $10 \%$ of cells overall, but appeared to preferentially stain the larger cells. HHV-8 was negative.

\section{Cytogenetic and molecular findings}

Fluorescence in situ hybridization on the lymph node showed no evidence of a BCL2 gene rearrangement. Molecular IGH assay, showed a strong peak in the FR3 region, consistent with a clonal B-cell process. Given the T-cell and histiocyte- rich background, TCR clonality studies were obtained and showed a TCR gamma gene rearrangement in the form of a peak in the V9_J13n23 region. This peak in the TCR gamma V9_J13n23 was in the least specific region and although this finding raised suspicion for an underlying clonal T-cell process, it did not lend specific support for the presence of a T-cell lymphoma. The TCR beta assay was negative.

On the basis of morphology, immunohistochemistry and molecular studies, a diagnosis of large B-cell lymphoma arising from follicular lymphoma, follicular pattern $40 \%$ and diffuse pattern $60 \%$ of the involved node, was rendered.

\section{Clinical follow-up}

At a follow-up visit in July 2013, the patient had further developed right upper quadrant discomfort, right lateral chest wall pain, left flank pain, and left lower quadrant abdominal pain with a mass under the left occiput. She had lost an additional four pounds but still denied night sweats, fever, or chills. At that visit there was a $2.5 \mathrm{~cm}$ soft cystic subcutaneous mass of the left occiput and bilateral cervical lymphadenopathy with the largest lymph node under the right mandible measuring $4.5 \mathrm{~cm}$. The staging bone marrow was uninvolved by lymphoma although the bone scan was considered to represent stage IV disease. The patient was given three cycles of immunochemotherapy consisting of rituximab, cyclophosphamide, doxorubicin, vincristine and prednisone (RCHOP). She suffered a recurrence in December 2013, which was documented by a needle core biopsy from the retroperitoneum demonstrating large B-cells associated with extensive necrosis and a high growth fraction. She is currently undergoing immunochemotherapy with rituximab, ifosfamide, carboplatin and etoposide (RICE), and is undergoing evaluation for stem cell transplantation. 
Citation: Nargund A, Eastep SJ, Warnke RA, Natkunam Y (2014) An Unusual Progression of Follicular Lymphoma Mimicking T-Cell and

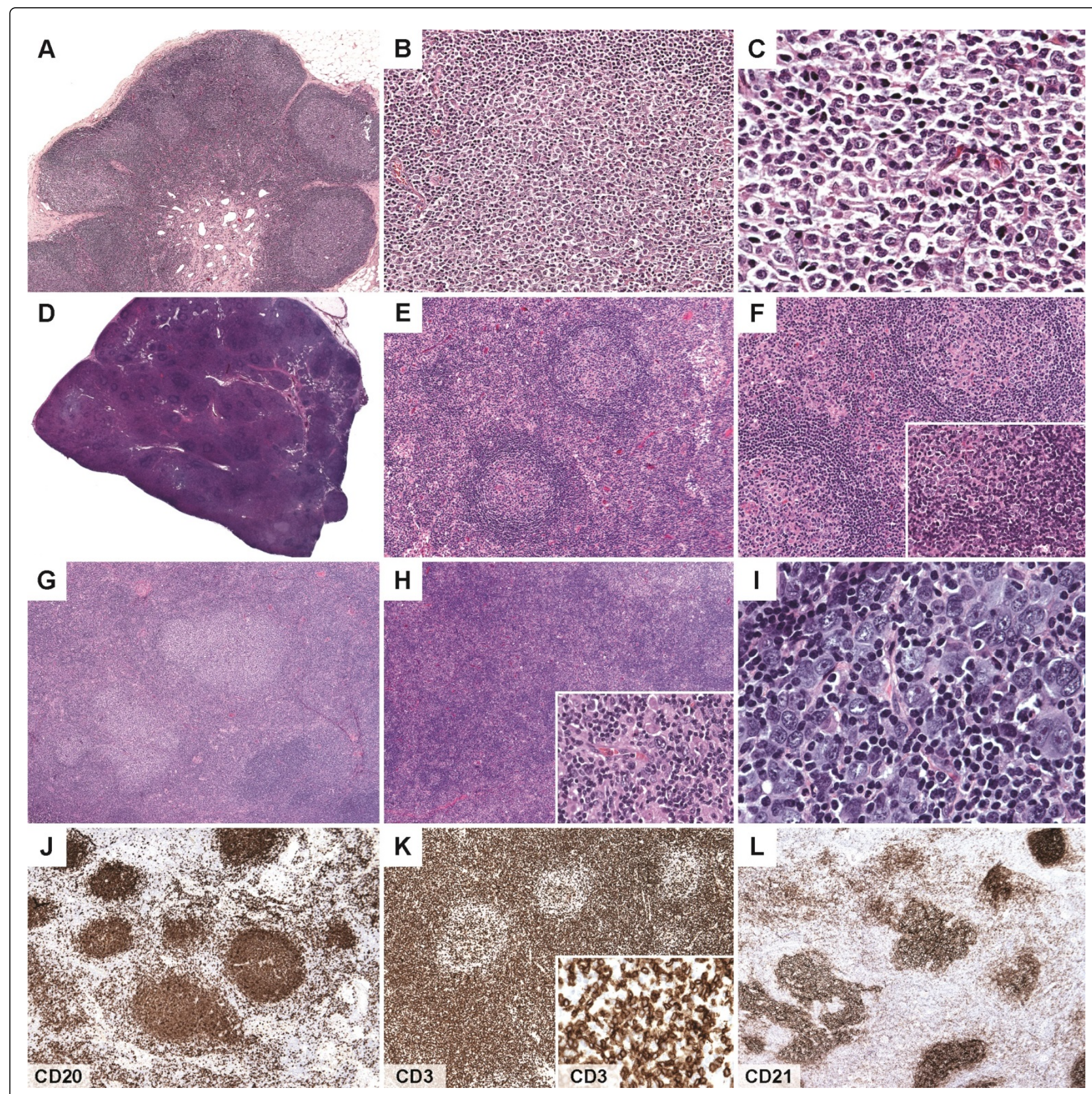

Figure 1: Comparison of histologic findings in 2001 and 2013 lymph nodes. Histologic sections of the lymph node from 2001 show well preserved overall architecture (A); The follicles show intact mantle zones and lack tangible body macrophages (B); A predominance of small cleaved cells are present within follicles(C); Histologic sections of the 2013 lymph node shows variably sized follicles (D); The follicles show intact mantle zones (E); The interfollicular areas are rich in polymorphous small and occasional large lymphoid cells and histiocytes (F); Some follicles show enlargement and lack of mantle zones $(\mathrm{G})$; These follicles also show increased numbers of large cells consistent with grade $3 \mathrm{~A}$ morphology $(\mathrm{H})$; A selected interfollicular area shows increased and atypical large cells, some resembling Hodgkin cells (I); Immunohistologic staining for CD20 highlights follicles as well as an interfollicular component (J); CD3 shows dense staining in the interfollicular/paracortical areas (K); CD21 highlights intact and expanded follicular dendritic cell meshworks (L). 
Citation: Nargund A, Eastep SJ, Warnke RA, Natkunam Y (2014) An Unusual Progression of Follicular Lymphoma Mimicking T-Cell and Histiocyte-Rich Large B-Cell Lymphoma . J Cytol Histol 5: 289. doi:10.4172/2157-7099.1000289



Figure 2: Immunohistologic findings in the 2013 lymph node. Atypical follicle with large cells within follicles and in mantle zones (A); these cells are highlighted by stains for OCT2 (B), HGAL (inset B), and BCL2 (C); a rare atypical follicles with confluence of large cells (D) highlighted by OCT2 (E) and BCL2 (F); interfollicular areas are studded with atypical large B-cells highlighted by CD20 in a TCRLBCL-like areas (G); Ki67 highlights a low proliferation in grade 1-2 follicular lymphoma (H); confluent focus of atypical large cells show staining for CD20 (I), and an increased growth fraction (J), but lack CD30 (K) and CD15 (L), confirming the presence of a diffuse large B-cell lymphoma.

\section{Discussion}

The histologic transformation of low-grade lymphomas, including follicular lymphoma, is a well-described phenomenon with an average risk of transformation of $20 \%$ at 5 years and $30 \%$ at 10 years. 1 Histological transformation to a more aggressive lymphoma, particularly, DLBCL, remains a critical event in the natural history of follicular, since it is usually associated with refractoriness to treatment, rapid clinical progression and adverse outcome [1-6]. The most frequent histologic transformation of FL includes a diffuse growth pattern and a predominance of large cells. In this report, we present an 
unusual case that underwent a rare histological transformation manifested by a T-cell and histiocyte-rich background and only occasional foci of confluent large B-cells. The spectrum of histologic findings in this case, especially given the absence of a prior history of lymphoma, raised a number of diagnostic challenges.

\begin{tabular}{|c|c|}
\hline Marker & Results \\
\hline CD20 & Positive in follicles and interfollicular atypical large cells \\
\hline CD79a & Positive in follicles and interfollicular atypical large cells \\
\hline BCL2 & $\begin{array}{l}\text { Positive in follicles and interfollicular atypical large cells; } \\
\text { positive in paracortical and interfolliculat T-cells }\end{array}$ \\
\hline PAX5 & Positive in follicles and interfollicular atypical large cells \\
\hline ОСТ2 & Positive in follicles and interfollicular atypical large cells \\
\hline HGAL & $\begin{array}{l}\text { Weakly positive in follicles and interfollicular atypical large } \\
\text { cells }\end{array}$ \\
\hline CD10 & Negative with appropriate internal control \\
\hline BCL6 & Negative with appropriate internal control \\
\hline LMO2 & Negative with appropriate internal control \\
\hline CD3 & Positive in paracortical and interfollicular small T-cells \\
\hline CD5 & Positive in paracortical and interfollicular small T-cells \\
\hline CD21 & $\begin{array}{l}\text { Positive in follicular dendritic cells; highlights FDC meshworks } \\
\text { in most follicles and only focal disruption in few follicles }\end{array}$ \\
\hline CD68 & $\begin{array}{l}\text { Positive in interfollicular histiocytic cells and highlights } \\
\text { increased numbers }\end{array}$ \\
\hline CD30 & Negative with appropriate internal control \\
\hline CD15 & Negative with appropriate internal control \\
\hline ALK1 & Negative with appropriate external control \\
\hline EMA & Negative with appropriate internal control \\
\hline PD-1 & Negative with appropriate internal control \\
\hline HHV8 & Negative with appropriate external control \\
\hline
\end{tabular}

Table 1: Immunohistologic profile of transformed follicular lymphoma.

At presentation, an antecedent history of lymphoma was not available. The overall nodular and diffuse architecture led to a broad differential diagnosis that included nodular lymphocyte predominant Hodgkin lymphoma, TCRLBCL as well as an underlying T-cell lymphoma such as peripheral $\mathrm{T}$-cell lymphoma or angioimmunoblastic T-cell lymphoma. The BCL2 stain was strongly positive within follicles and in diffuse areas and supported the diagnosis of a large B-cell lymphoma arising from follicular lymphoma. An HGAL stain was positive in the same areas, although it showed weak staining; however, in the absence of CD10 and BCL6, its expression in the neoplastic cells within follicles as well as in the diffuse infiltrate provided support for follicle center derivation. The large cells were dispersed within interfollicular areas and in small confluent foci. Although progression of FL to DLBCL is not unusual, the T-cell and histiocyte-rich interfollicular areas studded with large atypical B-cells in association with neoplastic BCL2-positive follicles, was extremely unusual. Upon further inquiry into the patient's prior history, an H\&E-stained section of the original lymph node from 2001 was retrieved. Although the findings were morphologically consistent with grade 1-2 follicular lymphoma, no immunohistochemistry or flow cytometry was available to provide support for that morphologic impression. It is also remarkable that this follicular lymphoma was clinically silent for twelve years, which is known to occur in a significant proportion of grade 1-2 follicular lymphoma [1].

FL is characterized by the presence of the $t(14 ; 18)$ chromosomal translocation, resulting in overexpression of the BCL2 protein; however, in a small proportion of FL, BCL2 rearrangements are not detected even with highly sensitive techniques but show overexpression of the BCL2 protein $[13,14]$ Our case reflected this small subset and was negative for $\mathrm{t}(14 ; 18)$ by FISH but expressed BCL2 protein by immunohistochemistry. An extra copy of chromosome 18 was also not detected, which is reported in similar cases. Given the Tcell rich background, we obtained TCR clonality studies, which ruled out a clonal $\mathrm{T}$-cell process and argued against the presence of an underlying T-cell lymphoma. In keeping with the adverse clinical outcome reported in transformed FL [1-6], this patient showed high stage disease that was refractory to treatment. Correlation with clinical findings was helpful in making the diagnosis of transformed FL.

We present a case of unusual progression of FL that had prominent T-cell and histiocyte-rich interfollicular areas containing large transformed cells, arising from an underlying FL. This case widens the histologic spectrum of FL transformation and also highlights the need for pathologists to be mindful that FL can transform to diffuse large B cell lymphoma in a morphologically unusual pattern. It is of importance to recognize this morphology in the context of FL progression, especially because the differential diagnosis includes disparate entities such as NLPHL, TCRLBCL and a large B-cell lymphoma arising in the background of an underlying $\mathrm{T}$-cell lymphoma, which require very different clinical management strategies and therapeutic and prognostic considerations.

\section{References}

1. Harris NL, Swerdlow SH, Jaffe ES, (2008) Follicular lymphoma. In Swerdlow SH CE, Harris NL. WHO Classification of Tumours of Haematopoietic and Lymphoid Tissues. Vol. 2 (4th Edition). IARC Press, Lyon, France

2. Tan D, Horning SJ, Hoppe RT, Levy R, Rosenberg SA, et al. (2013) Improvements in observed and relative survival in follicular grade 1-2 lymphoma during 4 decades: the Stanford University experience. Blood. 122: 981-987.

3. Link BK, Maurer MJ, Nowakowski GS, Ansell SM, Macon WR, et al. (2013) Rates and outcomes of follicular lymphoma transformation in the immunochemotherapy era: a report from the University of Iowa/ MayoClinic Specialized Program of Research Excellence Molecular Epidemiology Resource. J Clin Oncol 31: 3272-3278.

4. Bouska A, McKeithan TW, Deffenbacher KE, Lachel C, Wright GW et al. (2014) Genome-wide copy-number analyses reveal genomic abnormalities involved in transformation of follicular lymphoma. Blood 123: $1681-1690$.

5. Freedman A (2014) Follicular lymphoma: 2014 update on diagnosis and management. Am J Hematol 89: 429-436.

6. Guirguis HR, Cheung MC, Piliotis E, Spaner D, Berinstein NL, et al. (2014) Survival of patients with transformed lymphoma in the rituximab era. Ann Hematol 93: 1007-1014.

7. Come SE, Jaffe ES, Andersen JC, Mann RB, Johnson BL, et al. (1980) Non-Hodgkin's lymphomas in leukemic phase: clinicopathologic correlations. Am J Med 69: 667-674. 
Citation: Nargund A, Eastep SJ, Warnke RA, Natkunam Y (2014) An Unusual Progression of Follicular Lymphoma Mimicking T-Cell and Histiocyte-Rich Large B-Cell Lymphoma . J Cytol Histol 5: 289. doi:10.4172/2157-7099.1000289

Page 6 of 6

8. Natkunam Y, Soslow R, Matolcsy A, Dolezal M, Bhargava V, et al. (2004) Immunophenotypic and genotypic characterization of progression in follicular lymphomas. Appl Immunohistochem Mol Morphol 12: 97-104.

9. Natkunam Y, Warnke RA, Zehnder JL, Jones CD, Milatovich-Cherry A et al. (2000) Blastic/blastoid transformation of follicular lymphoma: immunohistologic and molecular analyses of five cases. Am J Surg Pathol 24: 525-534.

10. Weiss LM, Warnke RA (1985) Follicular lymphoma with blastic conversion: a report of two cases with confirmation by immunoperoxidase studies on bone marrow sections. Am J Clin Pathol 83: 681-686.

11. Horsman DE, Connors JM, Pantzar T, Gascoyne RD (2001) Analysis of secondary chromosomal alterations in 165 cases of follicular lymphoma with $\mathrm{t}(14 ; 18)$. Genes Chromosomes Cancer 30: 375-382.

12. Young KH, Xie Q, Zhou G, Eickhoff JC, Sanger WG, et al. (2008) Transformation of follicular lymphoma to precursor B-cell lymphoblastic lymphoma with c-myc gene rearrangement as a critical event. Am J Clin Pathol 129: 157-166.
13. Chiu A, Frizzera G, Mathew S, Hyjek EM, Chadburn A, et al. (2009) Diffuse blastoid B-cell lymphoma: a histologically aggressive variant of $\mathrm{t}(14 ; 18)$-negative follicular lymphoma. Mod Pathol 22: 1507-1517.

14. Horsman DE, Okamoto I, Ludkovski O, Le N, Harder L, et al. (2003) Follicular lymphoma lacking the $\mathrm{t}(14 ; 18)(\mathrm{q} 32 ; \mathrm{q} 21)$ : identification of two disease subtypes. Br J Haematol 120: 424-433.

15. Johnson NA, Savage KJ, Ludkovski O, Ben-Neriah S, Woods R, et al. (2009) Lymphomas with concurrent BCL2 and MYC translocations: the critical factors associated with survival. Blood 114: 2273-2279.

16. Gonzalez CL, Medeiros LJ, Jaffe ES (1991) Composite lymphoma. A clinicopathologic analysis of nine patients with Hodgkin's disease and Bcell non-Hodgkin's lymphoma. Am J Clin Pathol 96: 81-89.

17. Travis LB, Gonzalez CL, Hankey BF, Jaffe ES (1992) Hodgkin's disease following non-Hodgkin's lymphoma. Cancer 69: 2337-2342.

18. Batra R, Medeiros BC, Zehnder JL, Warnke RA, Natkunam Y (2012) Aggressive EBV-associated lymphoproliferative disorder: a prodrome to diffuse large B-cell lymphoma? Appl Immunohistochem Mol Morphol 20: $325-330$. 